\title{
Passively Q-switched Tm/Ho composite laser
}

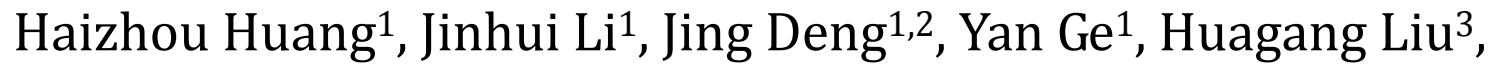 \\ Jianhong Huang1, Wen Weng1,2 and Wenxiong Lin ${ }^{1 *}$
}

\begin{abstract}
We explored Q-switching mechanism for the newly proposed Tm/Ho composite laser via developing a hybrid resonator for separating the intra-cavity Tm laser modulated by the saturable absorber (SA). With a Cr:ZnSe SA, successful passively Q-switching process with the maximum average output power of $474 \mathrm{~mW}$ and the shortest pulse width of $145 \mathrm{~ns}$ were obtained at the pulse repetition frequency of $7.14 \mathrm{kHz}$, where dual wavelength oscillation in both $2090 \mathrm{~nm}$ and 2097 $\mathrm{nm}$ was observed. This work provides an effective way for a direct laser diode (LD) pumped Q-switched Ho laser, which is compact and accessible. Furthermore, the current SA could be replaced by the 2D materials with broadband saturable absorption such as topological insulators or transition-metal dichalcogenides for seeking novel PQS lasers.
\end{abstract}

Keywords: Ho laser; Tm laser; Tm/Ho composite; passively Q-switched; Cr:ZnSe

Huang H Z, Li J H, Deng J, Ge Y, Liu H G et al. Passively Q-switched Tm/Ho composite laser. Opto-Electron Adv 3, 190031 (2020).

\section{Introduction}

Within the transparent window of atmosphere and the strong absorption band of water, Ho lasers have a series of important applications in fields such as surgeries, mid-infrared super-continuum, and lidar systems ${ }^{1-3}$, which have received more and more attentions. Particularly, pulsed Ho lasers with the wavelength around $2.1 \mu \mathrm{m}$ are efficient pump sources for mid-infrared frequency conversion toward the $3 \sim 12 \mu \mathrm{m}$ molecular fingerprint region ${ }^{4}$ via optical parameter oscillation (OPO) or differential frequency generation (DFG) $)^{5,6}$, owning to the wavelength farther away from absorption edge of the famous mid-infrared crystals ZPG and OP:GaAs than other Tm or Ho laser wavelengths ${ }^{7}$.

Due to the lack of absorption band of $\mathrm{Ho}^{3+}$ at $800 \mathrm{~nm}$, Ho lasers are commonly realized via the $\mathrm{Tm}$, Ho co-doped mechanism ${ }^{8}$ or intra-cavity pumping manner ${ }^{9}$ for directly utilizing the mature AlGaAs LDs. However, the above lasers have limitations: attributing to the cooperative up-conversion process between the $\mathrm{Tm}^{3+}$ and $\mathrm{Ho}^{3+}$ ions, the Tm, Ho co-doped lasers should be cooled by cryogenic devices for Watt-level operation. Rigorous calibration between the separately cooled Tm-doped and Ho-doped gain media should be maintained for the intra-cavity pumped Ho lasers, which complicated the system setup. Hence, with the development of $1.9 \mu \mathrm{m}$ lasers, such as Tm lasers or $1.9 \mu \mathrm{m} \mathrm{LD}$, in-band pumping became a major way to realize Ho lasers for the past two decades $^{10-12}$. However, the $1.9 \mu \mathrm{m}$ pump sources are more expensive than common AlGaAs LDs and the setup is bulky especially when using the Tm lasers due to the cascade pumping scheme ${ }^{13}$.

With the development of $2 \mu \mathrm{m}$ saturable absorbers, such as 1D materials (single wall carbon nanotube and gold nanorod) ${ }^{14,15}, 2 \mathrm{D}$ materials (graphene, topological insulators, and ternary chalcogenides) ${ }^{16-18}$, and transition metal ion-doped II-VI crystals $\left(\mathrm{Cr}^{2+}: \mathrm{ZnSe}, \mathrm{Cr}^{2+}: \mathrm{ZnS}\right)^{19,20}$, passively Q-switching (PQS) in $2 \mu \mathrm{m}$ Tm-doped and Ho-doped lasers has become a hot topic, which is characterized by compactness, easy implementation, and cost-saving compared with the actively Q-switching (AQS) manners. Among the above SAs, $\mathrm{Cr}^{2+}: \mathrm{ZnSe} / \mathrm{ZnS}$ crystals were demonstrated to be robust for achieving higher

\footnotetext{
${ }^{1}$ Key Laboratory of Optoelectronic Materials Chemistry and Physics, Fujian Institute of Research on the Structure of Matter, Chinese Academy of Sciences, Fuzhou 350002, China; ${ }^{2}$ University of Chinese Academy of Sciences, Beijing 100049, China; ${ }^{3}$ Department of Electrical and Computer Engineering, National University of Singapore, 4 Engineering Drive 3, 117576, Singapore.

*Correspondence: W X Lin, E-mail: wxlin@fjirsm.ac.cn

Received: 31 July 2019; Accepted: 17 September 2019; Published: 20 April 2020
} 
pulse energy and better PQS stability ${ }^{19}$, owing to the mature crystal growth process with fewer defects. Although both PQS and AQS were well demonstrated in in-band pumped and Tm, Ho co-doped Ho lasers ${ }^{12,21}$, Q-switching in intra-cavity pumped Ho lasers were limited due to the saturable effect in Ho-doped gain medium ${ }^{22,23}$, which led to a disorder pulse burst and the failure in Q-switching.

Recently, via integrating the Tm-doped and Ho-doped gain medium into a single bulk structure, the $\mathrm{Tm} / \mathrm{Ho}$ composite laser has been realized and demonstrated to meet the requirement of $2.1 \mu \mathrm{m}$ lasers in compactness, accessibility, and robustness ${ }^{24}$, which could be pumped by LDs with wavelength from $760 \mathrm{~nm}$ to $820 \mathrm{~nm}$ at broad room-temperatures ${ }^{25}$. However, pulse properties of such a Ho laser mechanism haven't been explored yet. On the other hand, saturable effect in both Tm-doped and Ho-doped regions of the composite gain medium will seriously challenge a successful Q-switching process due to the same resonant-pumping manner as the intra-cavity pumped Ho lasers. Hence, it is of significance to explore and realize Q-switching for the newly proposed $\mathrm{Tm} / \mathrm{Ho}$ composite laser.

In this paper, crystalline $\mathrm{Cr}^{2+}: \mathrm{ZnSe}$ crystal was selected as SA for exploring PQS properties of the Tm/Ho composite laser. Successful PQS process with the maximum output power of $474 \mathrm{~mW}$ and shortest pulse width of 145 ns was realized in a hybrid cavity structure, which could separate the intracavity $\mathrm{Tm}$ laser form Ho laser efficiently before modulated by the SA and in avoiding the cease in laser oscillation. The ideas here could also be adopted to realize PQS in the Tm/Ho composite lasers modulated by other SAs prepared with the 1D or 2D materials for seeking novel pulse Ho lasers.

\section{Experimental setup}

The schematic of the experiment is depicted in Fig. 1. A $\mathrm{Tm} / \mathrm{Ho}$ :YAG crystal integrated via diffusion bonding the 3.5 at.\% Tm:YAG and 0.6 at.\% Ho:YAG crystals into a single bulk structure was served as the gain medium. The composite gain medium had a dimension of $3 \mathrm{~mm} \times 3$ $\mathrm{mm} \times 14 \mathrm{~mm}$ with an $8 \mathrm{~mm}$ long $\mathrm{Tm}$-doped region, which was wrapped by indium foil and mounted into a copper heat sink for water cooling at $16{ }^{\circ} \mathrm{C}$. A concave-plano cavity with a physical length of $60 \mathrm{~mm}$ was applied to make sure the smallest cavity mode size on the output coupler M2. M1 was a plano-concave mirror with radius of curvature of $100 \mathrm{~mm}$, which was coated anti-refection (AR, $T>97 \%$ ) at $750-850 \mathrm{~nm}$ and high-reflection (HR,
$R>99.5 \%)$ at $1.9-2.2 \mu \mathrm{m}$. M2 was a plano-plano output coupler, which was HR at emission band of the Tm laser $(R>99 \%$ at $1.9-2.02 \mu \mathrm{m})$ and had transmittance of $10 \%$ at Ho laser ( $T \approx 10 \%$ at $2.09-2.2 \mu \mathrm{m}$ ) respectively.

The $\mathrm{Cr}^{2+}: \mathrm{ZnSe}$ SA with the dimension of $5 \mathrm{~mm} \times 5$ $\mathrm{mm} \times 1.7 \mathrm{~mm}$ and initial transmittance of $92 \%$ at $2.1 \mu \mathrm{m}$ was mounted into a copper heat sink and placed close to M2 for tight focusing operation ${ }^{26}$. For separating the intra-cavity $\mathrm{Tm}$ laser from modulated by the SA, a fused silica spectral filter F1 AR coated at 2.09-2.12 $\mu \mathrm{m}(T>95 \%)$ and HR coated at $1.9-2.02 \mu \mathrm{m}(R>93 \%)$ were inserted inside the cavity to form a hybrid cavity structure. A fiber coupled $808 \mathrm{~nm}$ LD (NL-PPS50, Nligt Inc.) with a core diameter of $400 \mu \mathrm{m}$ and numerical aperture of 0.22 was applied as the pump source. L1 and L2 are identical plano-convex mirrors with a focus length of $40 \mathrm{~mm}$ for forming a pump waist radius of $213 \mu \mathrm{m}$ inside the composite gain medium, which was calculated by the $A B C D$ matrix. M3 is a dichromatic mirror applied for filtering the Ho laser from the $800 \mathrm{~nm}$ pump light. The average output power was measured by an Ophir power meter (30(150)A-LP1-18, Ophire Inc.), and the lasing wavelength by a mid-IR spectrum analyzer (771A-IR, Bristol Instruments Inc.). Pulse properties of PQS composite Ho laser were analyzed by a $2 \mathrm{GHz}$ bandwidth oscilloscope (MSO 2034, Tektronix Inc.) connected with an InGaAs detector (DET05D/M, Thorlabs Inc).

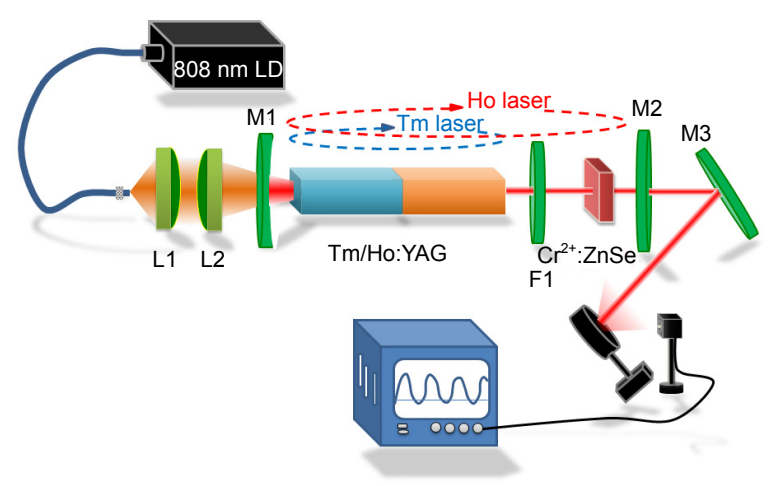

Fig. 1 | Layout of the PQS Tm/Ho composite laser.

\section{Resonator for PQS Tm/Ho composite laser}

According to the model for PQS lasers ${ }^{27}$, key threshold criterion for the $\mathrm{PQS}$ process is

$$
\alpha=\frac{\omega_{\mathrm{s}}^{2} \sigma_{\mathrm{gs}}}{\omega_{\mathrm{c}}^{2} \sigma}>\frac{n_{\mathrm{i}} / n_{\mathrm{th}}}{n_{\mathrm{i}} / n_{\mathrm{th}}-1},
$$

where $\sigma_{\mathrm{gs}}$ is ground state absorption cross section of the $\mathrm{SA}, \sigma$ is gain cross section of the Ho-doped region, $\omega_{\mathrm{s}}$ and $\omega_{\mathrm{c}}$ are cavity mode radii of the Ho laser on the composite 
gain medium and the SA, respectively. $n_{\mathrm{i}}$ and $n_{\mathrm{th}}$ are dimensionless parameters meeting

$$
\left\{\begin{array}{l}
n_{\mathrm{i}}=\ln \left(\frac{1}{T_{0}^{2}}\right)+\ln \left(\frac{1}{1-T_{\mathrm{OC}}}\right)+L \\
n_{\mathrm{th}}=\beta \ln \left(\frac{1}{T_{0}^{2}}\right)+\ln \left(\frac{1}{1-T_{\mathrm{OC}}}\right)+L
\end{array},\right.
$$

where $T_{0}$ is initial transmittance of the $\mathrm{SA}, T_{\mathrm{OC}}$ is transmittance of the output coupler, $L$ is the round-trip cavity loss, and $\beta$ is ratio between $\sigma_{\mathrm{gs}}$ and the excited state absorption cross section $\sigma_{\text {es }}$ of the SA.

In the concave-plano cavity for the $\mathrm{Tm} / \mathrm{Ho}$ composite laser described by $g$ parameters ${ }^{25}$

$$
\begin{gathered}
g_{i}=1-\frac{l_{0}}{R_{i}}-D\left(L_{j}+\frac{l_{\mathrm{r}}}{2 n}\right)\left[1-\left(L_{i}+\frac{l_{\mathrm{r}}}{2 n}\right) \frac{1}{R_{i}}\right], \\
i, j=1,2 \& i \neq j .
\end{gathered}
$$

Cavity mode waist $\omega_{\mathrm{s}}$ and $\omega_{\mathrm{c}}$ can be written as

$$
\begin{gathered}
\omega_{\mathrm{c}}=\omega_{\mathrm{s}} \sqrt{\left[1-\frac{1}{R_{1}}\left(l_{1}+\frac{l_{\mathrm{r}}}{2 n}\right)\right]^{2}+\left[\frac{1}{\left.l_{\mathrm{e}}\left(l_{1}+\frac{l_{\mathrm{r}}}{2 n}\right)\right]^{2} \frac{g_{1}\left(1-g_{1} g_{2}\right)}{g_{2}}}\right.}, \\
\omega_{\mathrm{s}}=\sqrt{\frac{\lambda_{l} l_{\mathrm{e}}}{\pi} \sqrt{\frac{g_{2}}{g_{1}\left(1-g_{1} g_{2}\right)}}}
\end{gathered}
$$

$$
\text { where } \quad l_{\mathrm{e}}=l_{1}+l_{2}+\frac{l_{\mathrm{r}}}{n}-D\left(l_{1}+\frac{l_{\mathrm{r}}}{2 n}\right)\left(l_{2}+\frac{l_{\mathrm{r}}}{2 n}\right)
$$

is the equivalent cavity length, $l_{0}=60 \mathrm{~mm}$ is length of the concave-plano cavity, $R_{i}$ is curvature of radius of mirror $M_{i}, l_{i}$ is distance between the gain medium and $M_{i} . l_{r}, n$, and $D$ are the length, refractive index, and thermal lens of the composite gain medium.

As described in Ref. ${ }^{28}, D$ is proportional to the absorbed pump power $P_{\text {abs }}$ and inverse proportional to the pump area as $D\left(P_{\text {abs }}\right)=C P_{\text {abs }} / \omega_{\mathrm{p}}^{2}$, where $C$ is $8.2893 \mathrm{~mm} / \mathrm{W}$ according to the calculation result in our previous work ${ }^{25}$. Hence, with other given cavity parameters, $\omega_{\mathrm{s}}$ and $\omega_{\mathrm{c}}$ are variables of $P_{\text {abs, }}$ as shown in Figs. 2(a) and 2(b). As denoted in Fig. 3, peak gain cross section $\sigma$ of the Ho-doped region is $1.4 \times 10^{-20} \mathrm{~cm}^{2}$. For the $\mathrm{Cr}^{2+}: \mathrm{ZnSe} \mathrm{SA}, \sigma_{\mathrm{gs}}$ and $\sigma_{\mathrm{es}}$ are $1.3 \times 10^{-19} \mathrm{~cm}^{2}$ and $0.9 \times 10^{-19} \mathrm{~cm}^{2}$ respectively ${ }^{29}$. Inserting values of $\sigma, \sigma_{\mathrm{gs}}$, and $\sigma_{\mathrm{es}}$ into Equation (2) and initializing $L$ as 0.03 , with the calculated $\omega_{\mathrm{s}}$ and $\omega_{\mathrm{c}}$, evolution in the key parameter $\alpha$ in Equation (1) with $P_{\text {abs }}$ is depicted in Fig. 2(c), where $\alpha_{\text {th }}=\frac{n_{\mathrm{i}}}{n_{\mathrm{th}}}\left(\frac{n_{\mathrm{i}}}{n_{\mathrm{th}}}-1\right)^{-1}$ denotes the threshold value. As shown in Fig. 2(c), the higher curvature radius $R_{1}$ leads to the lager stable region and the smaller $\alpha$, which is not suit for more stable PQS process according to threshold criterion. Although $R_{1}=75 \mathrm{~mm}$ contributes for the largest cavity mode size in the gain medium and the smallest mode size in SA for tight focusing among $R_{1}$ of $75 \mathrm{~mm}, 100 \mathrm{~mm}$ and $200 \mathrm{~mm}$, the shortest stable region denoted in Fig. 2(c) limits the achievable maximum output power for the PQS Ho laser. Therefore, $\mathrm{M}_{1}$ with $R_{1}$ of $100 \mathrm{~mm}$ was applied in the following experiment, which brings moderate $\alpha$ and higher unstable threshold at approximately $6.5 \mathrm{~W}$.
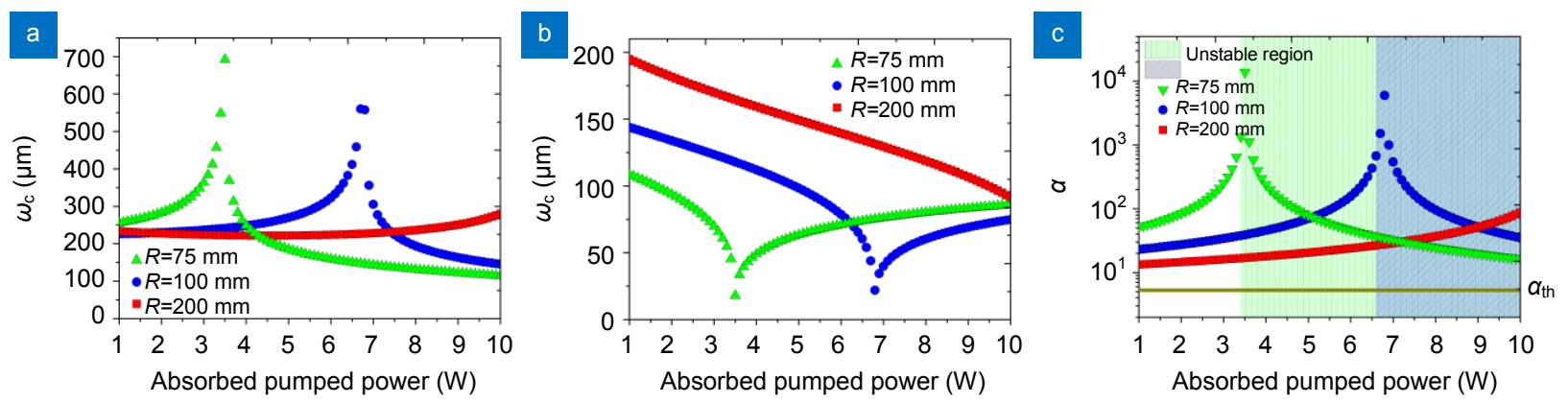

Fig. 2 ( $(\mathbf{a}, \mathbf{b})$ Evolutions in cavity mode sizes $\omega_{\mathrm{c}}$ and $\omega_{\mathrm{s}}$ with the absorbed LD power $P_{\mathrm{abs}}$ at different $\mathrm{M}_{1}$ curvature radii $\left(R_{1}=75 \mathrm{~mm}, 100 \mathrm{~mm}\right.$ and $200 \mathrm{~mm}$ ); (c) Evolution in key parameter $\alpha$ with $P_{\text {abs }}$, where the colorful areas denote corresponding unstable regions (green for $R_{1}=75 \mathrm{~mm}$, blue for $\left.R_{1}=100 \mathrm{~mm}\right), \alpha_{\mathrm{th}}$ is $\left(n_{i} / n_{\mathrm{th}}\right) /\left(n_{i} / n_{\mathrm{th}}-1\right)$ in Equation (1). Values of the parameters for the above calculation are summarized in Table 1.

Table 1 | Values of the parameters in Equations (1)-(5).

\begin{tabular}{c|c|c|c|c|c}
\hline Parameter & Value & Parameter & Value & Parameter & Value \\
\hline$\sigma_{\mathrm{gs}}$ & $1.3 \times 10^{-19} \mathrm{~cm}^{2}$, Ref. $^{29}$ & $\lambda$ & $2122 \mathrm{~nm}$ & $R_{1}$ & $75 \mathrm{~mm}, 100 \mathrm{~mm}, 200 \mathrm{~mm}$ \\
$\sigma$ & $1.4 \times 10^{-20} \mathrm{~cm}^{2}$ & $L$ & 0.03 & $I_{1}$ & $8 \mathrm{~mm}$ \\
\hline$\sigma_{\mathrm{es}}$ & $0.9 \times 10^{-19} \mathrm{~cm}^{2}$, Ref. $^{29}$ & $\omega_{\mathrm{p}}$ & $213 \mu \mathrm{m}$ & $I_{2}$ & $38 \mathrm{~mm}$ \\
\hline$C$ & $8.2893 \mathrm{~mm} / \mathrm{W}$, Ref. $^{25}$ & $T_{\mathrm{OC}}$ & $10 \%$ & $n$ & 1.82 \\
\hline$T_{0}$ & $92 \%$ & $R_{2}$ & Infinity & & \\
\hline
\end{tabular}

190031-3 


\section{Results and discussion}

\section{The start of PQS}

As shown in Fig. 3, emission bands of the intra-cavity Ho laser and Tm laser are within the designed initial transmittance curve of the SA. Due to the smaller gain cross section of $\mathrm{Tm}^{3+}$ ion compared with that of $\mathrm{Ho}^{3+}$ ion, oscillation in Tm laser was limited to sufficiently pump the Ho-doped region under both absorption losses from the Ho-doped region and the SA. Therefore, no oscillation in the PQS Tm/Ho composite laser was observed when directly inserted and adjusted the SA inside the laser cavity as normal PQS lasers.

As shown in Fig. 1, the spectral filter F1 was inserted into the cavity for preventing the Tm laser from being modulated and ceased by the SA and making sure the Ho laser oscillation. In the hybrid cavity consisted with M1, $\mathrm{M} 2$ and F1, characteristic pulse train of the free running Tm/Ho composite laser without SA is depicted in Fig. 4(a), which was irregular and unstable with time during the power scaling process. Stable PQS process occurred when inserting the SA into the hybrid cavity, where typical pulse train with the pulse repetition frequency (PRF) of $850 \mathrm{~Hz}$ and pulse width of $218 \mathrm{~ns}$ was shown in Fig. 4(b). Under the same absorbed LD power of $3.49 \mathrm{~W}$, pulse

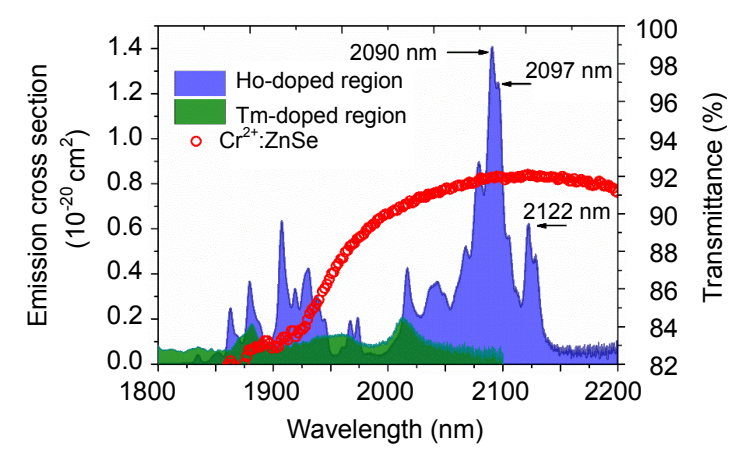

Fig. 3 | Emission cross section of the Tm-doped and Ho-doped regions and transmittance of the Cr:ZnSe SA.

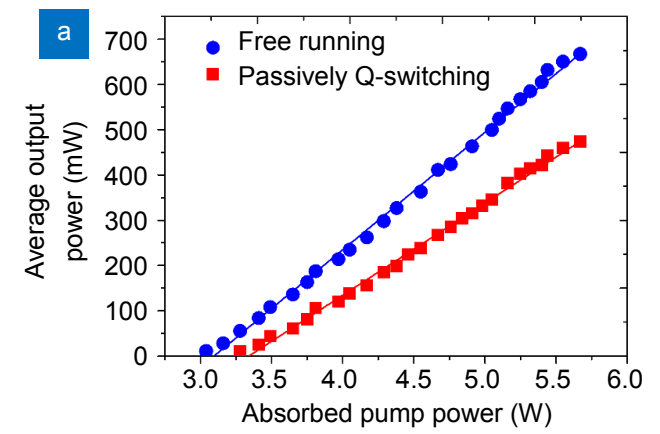

train in Fig. 4(b) was regular with higher signal intensity compared with that in Fig. 4(a).

\section{The laser properties}

Before inserting the Cr:ZnSe SA inside the hybrid cavity, free running properties were measured first with the maximum output power of $667 \mathrm{~mW}$ and slope efficiency of $25.9 \%$ considering the absorbed LD power. As shown in Fig. 5(a), using the SA, the maximum average power decreased to be $474 \mathrm{~mW}$ at an absorbed LD power of 5.67 W with a slope efficiency of $20.3 \%$ and the lasing threshold increased from $3.04 \mathrm{~W}$ to $3.28 \mathrm{~W}$ due to the insertion loss from the SA. Beam quality of the PQS Ho laser at the maximum output power was measured to be with $M_{x}{ }^{2}=1.22$ and $M_{y}{ }^{2}=1.15$ in the horizontal and vertical directions, respectively, via using a beam quality analyzer (Nanomode scan, Ophire Inc.), where the corresponding beam profile with a concentric $3 \mathrm{D}$ beam shape at the beam waist was depicted in Fig. 5(b).

Lasing wavelength for the free-running Ho laser was stabilized at approximately $2122.2 \mathrm{~nm}$ during the power scaling process, which is the same as that from a simple plano-concave cavity in our previous work ${ }^{25}$, where the characteristic spectrum at the maximum output power of $667 \mathrm{~mW}$ was shown in Fig. 6(a). During the PQS process,

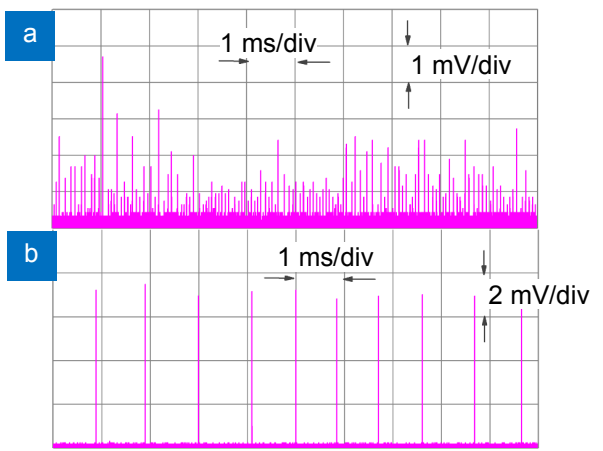

Fig. 4 | Comparison in pulse trains from the hybrid cavity before (a) and after (b) using the $\mathrm{Cr}^{2+}: \mathrm{ZnSe}$.

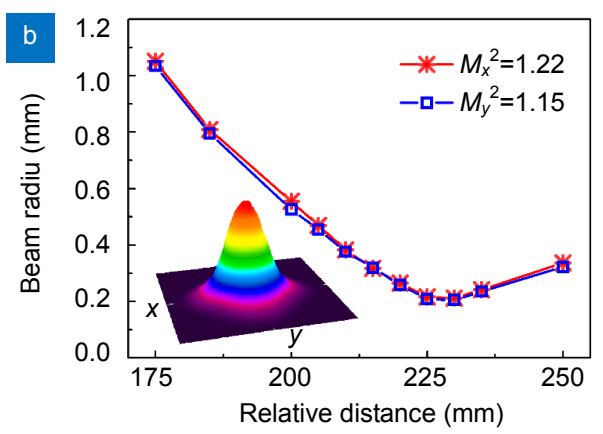

Fig. 5 | (a) Average output powers of the free-running Ho laser and PQS Ho laser versus absorbed pump power. (b) Beam profile of the PQS composite Ho laser at the maximum output power of $474 \mathrm{~mW}$ (Inset: 3D beam profile at the beam waist). 
the wavelength at $2090 \mathrm{~nm}$ oscillated first (Fig. 6(b)) before scaling up the output power above $200 \mathrm{~mW}$, where dual wavelength oscillation occurred at both $2090 \mathrm{~nm}$ and $2097 \mathrm{~nm}$ as shown in Fig. 6(c). Figure 6(d) illustrates the evolutions in Ho laser wavelengths at different output powers operating at free-running and PQS processes respectively. The measured Ho laser wavelengths were corresponding to the emission peaks of the Ho-doped region in Fig. 3, which switched from $2122 \mathrm{~nm}$ to shorter wavelengths due to the introduced cavity loss from the $\mathrm{SA}^{30}$.

Evolution in PRF and pulse width for the PQS composite Ho laser were measured and depicted in Fig. 7. Increasing the average output power from $20 \mathrm{~mW}$ to 474 $\mathrm{mW}$, PRF increased from $120 \mathrm{~Hz}$ to $7.14 \mathrm{kHz}$ with the pulse width decreased from 229 ns to 145 ns correspondingly. Typical pulse train at the maximum PRF of 7.14 $\mathrm{kHz}$ was depicted in Fig. 8(a). Figure 8(b) shows the de- tail view of typical pulse profile from Fig. 8(a), which has a pulse width of $145 \mathrm{~ns}$ and corresponds to a pulse energy and a peak power of $66 \mu \mathrm{J}$ and $458 \mathrm{~W}$, respectively.

\section{Conclusions}

In conclusion, we have demonstrated Q-switching properties of the recently proposed Tm/Ho composite laser, which met failure when following the traditional PQS manner due to the introduced modulation losses in both the intra-cavity Tm laser and Ho laser. A concave-plano hybrid resonator was designed for realizing a successful PQS process through filtering the intra-cavity Tm laser from Ho laser efficiently, where the maximum output power of $474 \mathrm{~mW}$ with a pulse width of $145 \mathrm{~ns}$ and a pulse energy of $66 \mu \mathrm{J}$ at PRF of $7.14 \mathrm{kHz}$ was obtained. Through paving a way for achieving Q-switching in the Tm/Ho composite mechanism, this work tries to provide
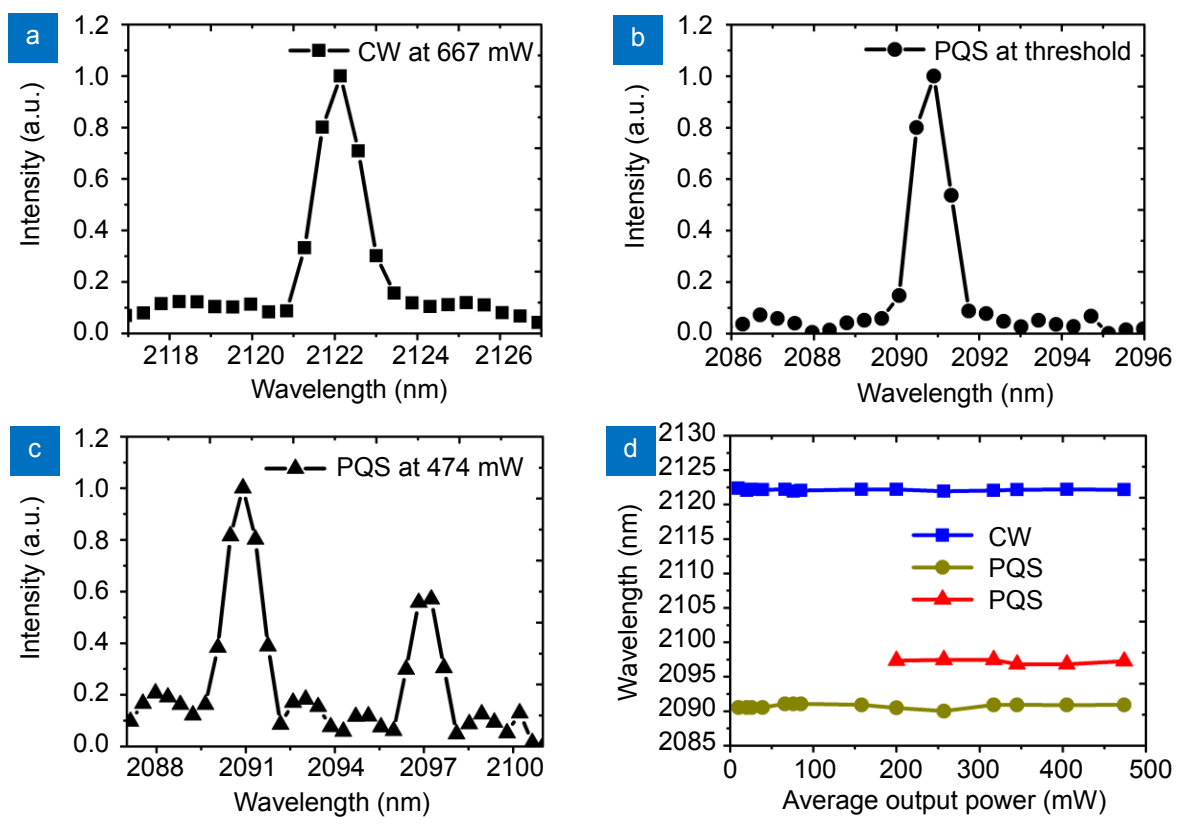

Fig. 6 | Wavelength properties of the Tm/Ho composite laser: (a) at the maximum free-running (CW) laser power; (b) at the threshold PQS laser power of $20 \mathrm{~mW}$; (c) at the maximum PQS laser power; (d) the measured peak wavelengths at different average output power.

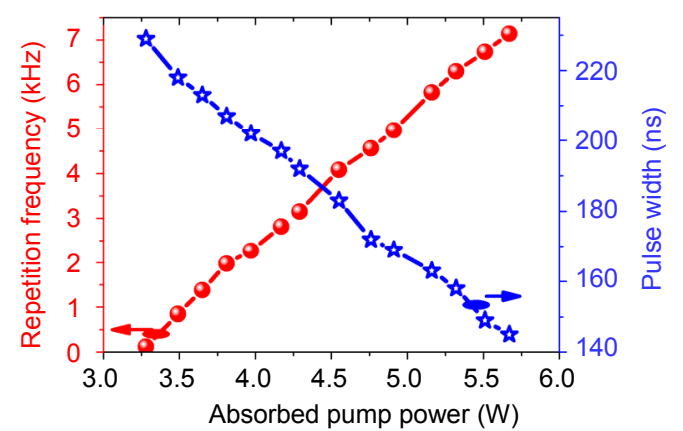

Fig. 7 | Evolutions in the pule repetition frequency and pulse width with the absorbed LD power.

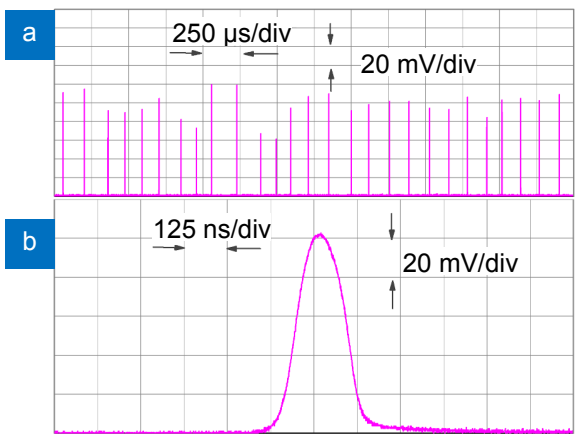

Fig. 8 | (a) Typical pules train with PRF of $7.14 \mathrm{kHz}$ at the maximum PQS output power. (b) Detail view of the shortest pulse with a width of $145 \mathrm{~ns}$ at the maximum output power. 
a compact and accessible pulse Ho laser which facilitates the direct use of common AlGaAs LDs. Moreover, the current SA can be replaced by the popular 2D materials, such as topological insulator, ternary chalcogenides, or graphene for exploring novel PQS Tm/Ho composite Ho lasers.

\section{References}

1. Hein S, Petzold R, Schoenthaler M, Wetterauer U, Miernik A. Thermal effects of Ho: YAG laser lithotripsy: real-time evaluation in an in vitro model. World J Urol 36, 1469-1475 (2018).

2. Zhang J W, Fai Mak K, Nagl N, Seidel M, Bauer D et al. Multi-mW, few-cycle mid-infrared continuum spanning from 500 to $2250 \mathrm{~cm}^{-1}$. Light Sci Appl 7, 17180 (2018).

3. Mizutani K, Ishii S, Aoki M, Iwai H, Otsuka R et al. $2 \mu \mathrm{m}$ Doppler wind lidar with a Tm:fiber-laser-pumped Ho:YLF laser. Opt Lett 43, 202-205 (2018).

4. Schliesser A, Picque N, Hansch T W. Mid-infrared frequency combs. Nat Photonics 6, 440-449 (2012).

5. Kanai T, Malevich P, Kangaparambil S S, Ishida K, Mizui M et al. Parametric amplification of $100 \mathrm{fs}$ mid-infrared pulses in $\mathrm{ZnGeP}_{2}$ driven by a Ho:YAG chirped-pulse amplifier. Opt Lett $\mathbf{4 2}$, 683-686 (2017).

6. Hemming A, Richards J, Davidson A, Carmody N, Bennetts S et al. $99 \mathrm{~W}$ mid-IR operation of a ZGP OPO at $25 \%$ duty cycle. Opt Express 21, 10062-10069 (2013).

7. Schunemann P G. New nonlinear crystals for the mid-infrared. In Nonlinear Optics 2017 (Optical Society of America, 2017); http://doi.org/10.1364/NLO.2017.NTu2A.1.

8. Budni P A, Pomeranz L A, Lemons M L, Schunemann P G, Pollak T M et al. 10W Mid-IR holmium pumped $\mathrm{ZnGeP} 2$ OPO. In Advanced Solid State Lasers 1998 (Optical Society of America, 1998); http://doi.org/10.1364/ASSL.1998.FC1.

9. Bollig C, Hayward R A, Clarkson W A, Hanna D C, 2-W Ho:YAG laser intracavity pumped by a diode-pumped Tm:YAG laser. Opt Lett 23, 1757-1759 (1998).

10. Budni P A, Pomeranz L A, Miller C A, Dygan B K, Lemons $M L$ et al. CW and Q-switched Ho:YAG pumped by Tm:YALO. In Advanced Solid State Lasers 1998 (Optical Society of America, 1998); http://doi.org/10.1364/ASSL.1998.ML4

11. Chen $\mathrm{H}$, Shen $D$ Y, Zhang J, Yang H, Tang D Y et al. In-band pumped highly efficient Ho:YAG ceramic laser with $21 \mathrm{~W}$ output power at $2097 \mathrm{~nm}$. Opt Lett 36, 1575-1577 (2011).

12. Zhang $Y X$, Gao $C Q$, Wang $Q, N a Q X$, Zhang $M$ et al. Single-frequency, injection-seeded $Q$-switched Ho:YAG ceramic laser pumped by a $1.91 \mu \mathrm{m}$ fiber-coupled LD. Opt Express 24, 27805-27811 (2016).

13. Lamrini $S$, Koopmann $P$, Schäfer $M$, Scholle K, Fuhrberg $P$. Directly diode-pumped high-energy Ho:YAG oscillator. Opt Lett 37, 515-517 (2012).

14. Chernysheva M, Mou C B, Arif R, AlAraimi M, Rümmeli M et al. High power Q-switched thulium doped fibre laser using carbon nanotube polymer composite saturable absorber. Sci Rep 6 , 24220 (2016).

15. Huang $H$ T, Li M, Liu P, Jin L, Wang $H$ et al. Gold nanorods as the saturable absorber for a diode-pumped nanosecond Q-switched $2 \mu \mathrm{m}$ solid-state laser. Opt Lett 41, 2700-2703 (2016).

16. Zhao T, Wang $\mathrm{Y}$, Chen $H$, Shen $D$ Y. Graphene passively
Q-switched Ho:YAG ceramic laser. Appl Phys B 116, 947-950 (2014).

17. Liu X, Yang K, Zhao S, Li T, Qiao W et al. High-power passively $Q$-switched $2 \mu \mathrm{m}$ all-solid-state laser based on a $\mathrm{Bi}_{2} \mathrm{Te}_{3}$ saturable absorber. Photonics Res 5, 461-466 (2017).

18. Yan B Z, Zhang B T, He J L, Nie H K, Li G R et al. Ternary chalcogenide $\mathrm{Ta}_{2} \mathrm{NiS}_{5}$ as a saturable absorber for a $1.9 \mu \mathrm{m}$ passively Q-switched bulk laser. Opt Lett 44, 451-454 (2019).

19. Cole B, Goldberg L. Highly efficient passively Q-switched Tm:YAP laser using a Cr:ZnS saturable absorber. Opt Lett $\mathbf{4 2}$, 2259-2262 (2017).

20. Lan J L, Xu B, Zhang Y Z, Xu H Y, Cai Z P et al. Tunable and passively Q-switched laser operation of $\mathrm{Tm}: \mathrm{CaYAlO}_{4}$ between $1848 \mathrm{~nm}$ and $1876 \mathrm{~nm}$. Opt Laser Technol 109, 33-38 (2019).

21. Li L J, Yang X N, Zhou L, Xie W Q, Wang $Y L$ et al. Active/passive $Q$-switching operation of $2 \mu \mathrm{m}$ Tm,Ho:YAP laser with an acousto-optical $Q$-switch/MoS 2 saturable absorber mirror Photonics Res 6, 614-619 (2018).

22. Schellhorn M, Hirth A, Kieleck C. Ho:YAG laser intracavity pumped by a diode-pumped Tm:YLF laser. Opt Lett $\mathbf{2 8}$, 1933-1935 (2003).

23. Yang $X$ F, Huang $H$ T, Shen D Y, Zhu $H$ Y, Tang D Y. $2.1 \mu \mathrm{m}$ Ho:LuAG ceramic laser intracavity pumped by a diode-pumped Tm:YAG laser. Chin Opt Lett 12, 121405 (2014).

24. Huang $\mathrm{H} Z$, Huang J H, Ge Y, Zheng H, Weng $W$ et al. $2.1 \mu \mathrm{m}$ composite Tm/Ho:YAG laser. Opt Lett 43, 1271-1274 (2018).

25. Huang H Z, Deng J, Ge Y, Li J H, Huang J H et al. Direct 800 $\mathrm{nm}$ diode-pumped Holmium laser with broad pump wavelength range and temperature adaptability. Opt Express 27, 13492-13502 (2019).

26. Huang $Y$ J, Huang $Y P$, Chiang $P$, Liang $H ~ C$, Su $K$ W et al. High-power passively Q-switched Nd:YVO $\mathrm{YV}_{4}$ UV laser at $355 \mathrm{~nm}$. Appl Phys B 106, 893-898 (2012).

27. Tuan $P H$, Chang $C C$, Chang $F L$, Lee $C Y$, Sung $C L$ et al. Modelling end-pumped passively Q-switched Nd-doped crystal lasers: manifestation by a $\mathrm{Nd}: \mathrm{YVO}_{4} / \mathrm{Cr}^{4+}: \mathrm{YAG}$ system with a concave-convex resonator. Opt Express 25, 1710-1722 (2017).

28. Chang Y T, Huang Y P, Su K W, Chen Y F. Comparison of thermal lensing effects between single-end and double-end diffusion-bonded Nd:YVO $\mathrm{Y}_{4}$ crystals for ${ }^{4} \mathrm{~F}_{3 / 2} \rightarrow{ }^{4} \mathrm{I}_{11 / 2}$ and ${ }^{4} \mathrm{~F}_{3 / 2} \rightarrow{ }^{4} \mathrm{I}_{13 / 2}$ transitions. Opt Express 16, 21155-21160 (2008).

29. Podlipensky A V, Shcherbitsky V G, Kuleshov N V, Levchenko V I, Yakimovich $V \mathrm{~N}$ et al. $1 \mathrm{~W}$ continuous-wave laser generation and excited state absorption measurements in $\mathrm{Cr}^{2+}: \mathrm{ZnSe}$. In Advanced Solid State Lasers 2000 (Optical Society of America, 2000); http://doi.org/10.1364/ASSL.2000.MC7.

30. Barnes N P, Amzajerdian F, Reichle D J, Carrion W A, Busch G $E$ et al. Diode pumped Ho:YAG and Ho:LuAG lasers, Q-switching and second harmonic generation. Appl Phys B 103, 57-66 (2011).

\section{Acknowledgements}

We are grateful for financial supports from National Key Research and Development Program of China (Grant No. 2017YFB1104500), Natural National Science Foundation of China (NSFC) (Grant No. 61875200), and China Postdoctoral Science Foundation (Grant No. 2018M642575)

\section{Competing interests}

The authors declare no competing financial interests. 